\title{
Highly Efficient Microwave-assisted Aminolysis of Epoxides in Water ${ }^{\dagger}$
}

\author{
Hua Zuo, ${ }^{\ddagger}, \#$ Zhu-Bo Li, ${ }^{\ddagger}$ Bao-Xiang Zhao, ${ }^{\S} *$ Jun-Ying Miao, ${ }^{\S}$ Li-juan Meng, ${ }^{\S}$ Kiwan Jang,, \\ Chuljin Ahn, Dong-Ha Lee, ${ }^{\text {, }}$ and Dong-Soo Shin ${ }^{\#, *}$ \\ "College of Pharmaceutical Sciences, Southwest University, Chongqing, 400716, China \\ $\S$ Institutes of Organic Chemistry and Developmental Biology, Shandong University, Jinan, 250100, China \\ ${ }^{*}$ E-mail: bxzhao@sdu.edu.cn \\ ${ }^{\#}$ Departments of Chemistry \& Physics, Changwon National University, Changwon, GN, 641-733, S. Korea \\ "E-mail:dsshin@changwon.ac.kr \\ 'Dongbu Advanced Research Institute, Dongbu Hannong Chemical Co., Ltd. Daejeon 305-708, S. Korea \\ Received March 11, 2011, Accepted April 1, 2011
}

Highly efficient and rapid aminolysis of epoxides with various amines in water under microwave irradiation in the absence of catalyst was developed. Chiral $\beta$-amino alcohols were formed in a short time with excellent yields.

Key Words : Microwave-assisted, Aminolysis, Epoxide, Water, Amino alcohol, Ring opening

\section{Introduction}

Chiral $\beta$-amino alcohols are very important organic compounds due to their use as intermediates in the synthesis of biologically active natural products and pharmaceuticals and medicinal chemistry. ${ }^{1}$ They were typically prepared from ring opening of epoxides by reacting with much excess amine at elevated temperature for long time. ${ }^{2}$ Since poor control of regioselectivity or some functional groups may be susceptible to high temperature, a variety of metallic catalysts ${ }^{3-24}$ and nonmetallic catalyst ${ }^{25-29}$ have been introduced for the cleavage of epoxides in recent years. Recent method without using catalyst in water for long reaction time has been reported. ${ }^{30}$

However, the use of expensive catalysts and toxic organic solvents, or long reaction time limited the application of aminolysis of epoxides. Take account of the advantages of microwave irradiation such as enhancement of the rate of reaction, higher yield, greater selectivity and decrease in reaction time, we herein present the first highly efficient microwave-assisted aminolysis of epoxide by the reaction of a variety of amines with epoxides in the absence of any catalyst in water. Since using only water instead of any organic solvents and microwave heating, it showed the great importance from the point of view of green chemistry and especially high efficiency. The reaction can also be performed at room temperature, while it takes more than 3 days to complete the reaction with very low yield.

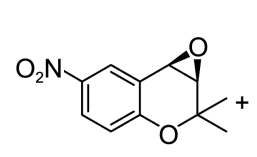

3

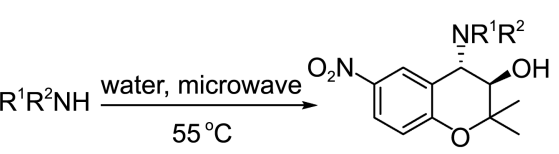

5
1
Scheme 1. Reaction of epoxide 3 with amines in water under microwave irradiation.

This paper is dedicated to Professor Eun Lee on the occasion of his honourable retirement.

\section{Results and Discussions}

Treatment of epoxide $\mathbf{3}$ or $\mathbf{4}$ with 1.2 equiv of substituted aryl piperazines afforded chiral 3-hydroxy-6-nitro-4-substituted piperazin-1-yl chromans $\mathbf{1}$ and $\mathbf{2}$ as asymmetric ring-opening product, respectively (Scheme 1).

These reactions proceeded smoothly in water under microwave irradiation with a very simple procedure without using any organic solvent or catalyst. The reaction is very easy because product isolation and purification is straightforward, requiring simple solvent (EtOAc) extraction and solvent evaporation or column chromatography in some cases.

Table 1. Synthesis of $\mathbf{1}$ by asymmetric ring opening reaction of epoxide, $\mathbf{3}$ with amines, $\mathbf{5}$ in water under microwave irradiation.

\begin{tabular}{|c|c|c|c|c|}
\hline Entry & Amine & Product & $\begin{array}{l}\text { Reaction time } \\
\text { (min) }\end{array}$ & $\begin{array}{l}\text { Yield } \\
(\%)^{a}\end{array}$ \\
\hline 1 & & $1 a$ & 18 & 85 \\
\hline 2 & & $1 b$ & 20 & 82 \\
\hline 3 & & 1c & 17 & 79 \\
\hline 4 & & $1 d$ & 22 & 82 \\
\hline 5 & & $1 \mathrm{e}$ & 18 & 83 \\
\hline 6 & & $1 f$ & 15 & 85 \\
\hline 7 & & $1 \mathrm{~g}$ & 18 & 90 \\
\hline
\end{tabular}

${ }^{a}$ lsolated yield. 
Table 2. Synthesis of $\mathbf{2}$ by asymmetric ring opening reaction of epoxide, $\mathbf{4}$ with amines, $\mathbf{5}$ in water under microwave irradiation.

\begin{tabular}{|c|c|c|c|c|}
\hline Entry & Amine & Product & $\begin{array}{l}\text { Reaction time } \\
\text { (min) }\end{array}$ & $\begin{array}{l}\text { Yielc } \\
(\%)^{a}\end{array}$ \\
\hline 1 & & $2 a$ & 23 & 85 \\
\hline 2 & & $2 \mathrm{~b}$ & 18 & 83 \\
\hline 3 & & $2 c$ & 19 & 80 \\
\hline 4 & & 2d & 19 & 81 \\
\hline 5 & & $2 e$ & 20 & 79 \\
\hline 6 & & $2 f$ & 21 & 76 \\
\hline 7 & & $2 \mathrm{~g}$ & 18 & 82 \\
\hline
\end{tabular}

${ }^{a}$ Isolated yield.

Moreover, the yields of all the reactions were excellent as shown in Table 1 and Table 2. It gave a more straightforward way compared to other methods involved several steps and catalyst reported in the literature. $^{31}$

The data clearly show that the reaction of different amines with epoxides in water under microwave irradiation gives corresponding chiral $\beta$-amino alcohols within minutes instead of hours or days. The $\beta$-amino alcohols thus obtained gave satisfactory IR, ${ }^{1} \mathrm{H} \mathrm{NMR},{ }^{13} \mathrm{C}$ NMR, mass spectral data and optical rotation value. This method is superior to other existing methods in that it forms single product under moderate temperature in very high yields (76-90\%) and very short time (15-23 $\mathrm{min}$ ) in water.

The formation of epoxide $\mathbf{3}$ was through five steps illustrated in Scheme 3. ${ }^{32}$ First it involved the nitration of 2hydroxyacetophenone to give 2-hydroxy-5-nitroacetophenone 7. Then the chromanone 8 was formed by reacting 7 with acetone catalyzed by pyrrolidine in refluxing toluene. Compound 9 was obtained by the reduction of $\mathbf{8}$ by $\mathrm{NaBH}_{4}$. Finally epoxide 3 was afforded via the epoxidation of $2 H$-chromene 10, which was obtained through dehydration of 9 , by $(R, R)$ Jacobson's catalyst. Similarly, epoxide $\mathbf{4}$ was formed by catalysis of $(S, S)$ Jacobson's catalyst. Yields were high in all steps.

Reaction conditions: (a) fuming $\mathrm{HNO}_{3} / \mathrm{H}_{2} \mathrm{SO}_{4}$; (b) acetone,

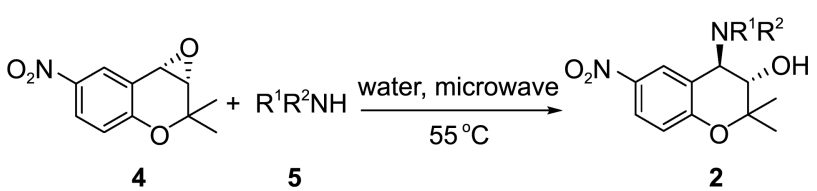

Scheme 2. Reaction of epoxide 4 with amines in water under microwave irradiation.

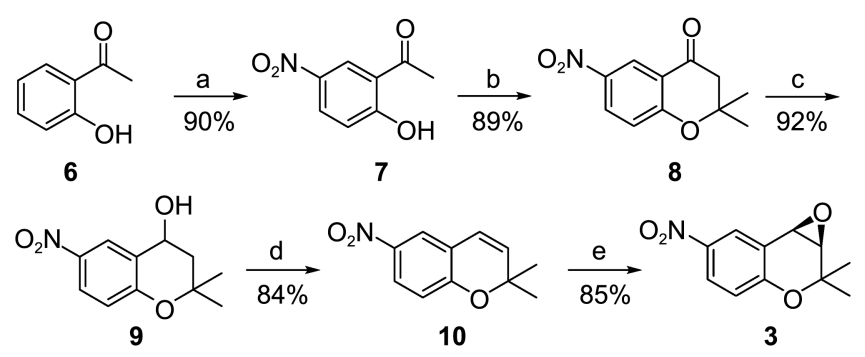

Scheme 3. Preparation of epoxide 3 via nitration, ring cyclization, reduction, dehydration and epoxidation.

pyrrolidine, toluene, reflux; (c) $\mathrm{NaBH}_{4}, \mathrm{MeOH},-5{ }^{\circ} \mathrm{C}$-rt.; (d) $p$ Toluenesulfonic acid monohydrate, toluene, reflux; (e) $(R, R)-$ Jacobsen's catalyst, $\mathrm{NaOCl}, \mathrm{Na}_{2} \mathrm{HPO}_{4} \cdot 12 \mathrm{H}_{2} \mathrm{O}, \mathrm{CH}_{2} \mathrm{Cl}_{2}, \mathrm{H}_{2} \mathrm{O}$, $-5^{\circ} \mathrm{C}-\mathrm{rt}$.

\section{Conclusions}

In summary, the present study is the first highly efficient and practical method for the preparation of $\beta$-amino alcohols in water by microwave irradiation. This method offers high yields, very short reaction time, simple procedure and environmentally friendly conditions without any catalyst and organic solvents, compared with the conventional existing methods. The determination of biological activities of these products is underway.

\section{Experimental Section}

General. ${ }^{1} \mathrm{H}$ and ${ }^{13} \mathrm{C}$ NMR spectra were recorded in $\mathrm{CDCl}_{3}$ (400 MHz for ${ }^{1} \mathrm{H}$ and $100 \mathrm{MHz}$ for ${ }^{13} \mathrm{C}$, respectively) with TMS as the internal reference on Bruker Advance 400 FT spectrometer. Chemicals shifts were reported in parts per million. Mass spectra (MS) were measured by the EI method. The specific rotation values were determined by a Digital polarimeter (DIP-1000, Jasco). Melting points are uncorrected. Silica gel (70-230 mesh) was used for flash column chromatography. All the reactions were monitored by TLC using $0.25 \mathrm{~mm}$ silica gel plates (Merck 60F-254) with UV indicator. All microwave-assisted reactions were carried out on $1.5 \mathrm{KW}$ Microwave heating device (KMIC). The microwaveassisted reaction time is the hold time at the final temperature. All chemicals were used as delivered from SigmaAldrich or Acros.

General Procedure for the Synthesis of Compound 1 and 2. Epoxide ( $1.81 \mathrm{mmol}, 1.0$ equiv) and amine (2.35 mmol, 1.2 equiv) were mixed in water and placed in a round-bottomed flask. The mixture was subjected to microwave irradiation $\left(1 \mathrm{KW}, 55^{\circ} \mathrm{C}\right)$ for $15-23 \mathrm{~min}$. The reaction was monitored with TLC. And then the reaction mixture was extracted with ethyl acetate and dried over $\mathrm{MgSO}_{4}$. The solvent was removed under reduced pressure to give the chiral $\beta$-amino alcohols. In some cases, further purification by column chromatography on silica gel was carried out.

(3R,4S)-2,2-Dimethyl-6-nitro-4-(4-phenylpiperazin-1yl)chroman-3-ol (1a): A light-yellow solid, mp 155-157 ${ }^{\circ} \mathrm{C}$. 
$[\alpha]_{D}^{20}-155.8\left(c 1.0, \mathrm{CH}_{2} \mathrm{Cl}_{2}\right)$; IR (KBr) $v / \mathrm{cm}^{-1}: 3430,3030$, $2977,2940,2831,1601,1583,1514,1478,1451,1427$, $1383,1332,1271,1238,1170,1136,1071,1013,998,943$, 921, 840, 751, 696; ${ }^{1} \mathrm{H}$ NMR (400 MHz, $\left.\mathrm{CDCl}_{3}\right) \delta 1.24$ (s, $3 \mathrm{H}), 1.52(\mathrm{~s}, 3 \mathrm{H}), 2.74(\mathrm{~s}, 1 \mathrm{H}), 2.99-3.08(\mathrm{~m}, 4 \mathrm{H}), 3.19-3.26$ $(\mathrm{m}, 4 \mathrm{H}), 3.79-3.85(\mathrm{~m}, 2 \mathrm{H}), 6.86-6.96(\mathrm{~m}, 4 \mathrm{H}), 7.26-7.30$ $(\mathrm{m}, 2 \mathrm{H}), 8.05(\mathrm{dd}, J=9.2,2.8 \mathrm{~Hz}, 1 \mathrm{H}), 8.49(\mathrm{~d}, J=2.8 \mathrm{~Hz}$, $1 \mathrm{H}) ;{ }^{13} \mathrm{C}$ NMR $\left(100 \mathrm{MHz}, \mathrm{CDCl}_{3}\right) \delta 18.7,26.7,49.8,50.7$, 63.1, 70.3, 79.9, 116.5, 118.0, 120.2, 122.8, 124.7, 124.9, 129.2, 141.4, 151.4, 159.4; MS (EI) $m / z: 383\left(\mathrm{M}^{+}, 20 \%\right), 312$ (17), 161 (10), 132 (100), 118 (21), 104 (16), 77 (8), 56 (10).

(3R,4S)-4-(4-(2-Fluorophenyl)piperazin-1-yl)-2,2-dimethyl6-nitrochroman-3-ol (1b): A light-yellow solid, mp 82-85 ${ }^{\circ} \mathrm{C} ;[\alpha]_{D}^{20}-123.6\left(c \quad 1.0, \mathrm{CH}_{2} \mathrm{Cl}_{2}\right)$; IR $(\mathrm{KBr}) v / \mathrm{cm}^{-1}: 3433$, 3076, 2977, 2937, 2830, 1614, 1584, 1518, 1500, 1478, $1453,1428,1379,1340,1271,1238,1207,1168,1137$, $1075,1013,996,944,926,839,809,750,695 ;{ }^{1} \mathrm{H}$ NMR $\left(400 \mathrm{MHz}, \mathrm{CDCl}_{3}\right) \delta 1.25(\mathrm{~s}, 3 \mathrm{H}), 1.54(\mathrm{~s}, 3 \mathrm{H}), 2.58(\mathrm{~d}, J=$ $2.4 \mathrm{~Hz}, 1 \mathrm{H}), 3.02-3.10(\mathrm{~m}, 4 \mathrm{H}), 3.12-3.19(\mathrm{~m}, 4 \mathrm{H}), 3.79(\mathrm{~d}, J$ $=10.0 \mathrm{~Hz}, 1 \mathrm{H}), 3.87(\mathrm{dd}, J=10.0,2.4 \mathrm{~Hz}, 2 \mathrm{H}), 6.87(\mathrm{~d}, J=$ $9.2 \mathrm{~Hz}, 1 \mathrm{H}), 6.93-7.10(\mathrm{~m}, 4 \mathrm{H}), 8.05(\mathrm{dd}, J=9.2,2.4 \mathrm{~Hz}$, $1 \mathrm{H}), 8.51(\mathrm{~d}, J=2.0 \mathrm{~Hz}, 1 \mathrm{H}) ;{ }^{13} \mathrm{C} \mathrm{NMR}\left(100 \mathrm{MHz}, \mathrm{CDCl}_{3}\right)$ $\delta$ 18.7, 26.7, 49.8, 50.7, 63.1, 70.3, 79.9, $116.2\left(\mathrm{~d}, J_{\mathrm{CF}}=18.7\right.$ $\mathrm{Hz}), 118.0,119.2,122.7\left(\mathrm{~d}, J_{\mathrm{CF}}=7.7 \mathrm{~Hz}\right), 122.9,124.5$, $124.8\left(\mathrm{~d}, J_{\mathrm{CF}}=21.0 \mathrm{~Hz}\right), 140.0\left(\mathrm{~d}, J_{\mathrm{CF}}=8.7 \mathrm{~Hz}\right), 141.5$, 154.5, 157.0, 159.4; MS (EI) $m / z$ : $383\left(\mathrm{M}^{+}, 20 \%\right), 312$ (17), 161 (10), 132 (100), 118 (21), 104 (16), 77 (8), 56 (10).

(3R,4S)-4-(4-(4-Fluorophenyl)piperazin-1-yl)-2,2-dimethyl6-nitrochroman-3-ol (1c): A light-yellow solid, mp 154$156^{\circ} \mathrm{C} ;[\alpha]_{D}^{20}-108.0\left(c 1.0, \mathrm{CH}_{2} \mathrm{Cl}_{2}\right)$; IR $(\mathrm{KBr}) v / \mathrm{cm}^{-1}: 3462$, $3096,3054,2976,2944,2899,2880,2837,1612,1584$, $1512,1483,1456,1427,1412,1384,1370,1338,1319$, $1283,1263,1233,1221,1171,1137,1094,1064,1009,985$, 948, 916, 837, 820, 813, 762, 749, 718, 707, 688; ${ }^{1} \mathrm{H}$ NMR $\left(400 \mathrm{MHz}, \mathrm{CDCl}_{3}\right) \delta 1.25(\mathrm{~s}, 3 \mathrm{H}), 1.53(\mathrm{~s}, 3 \mathrm{H}), 2.63(\mathrm{~d}, J=$ $2.8 \mathrm{~Hz}, 1 \mathrm{H}), 3.00-3.09(\mathrm{~m}, 4 \mathrm{H}), 3.11-3.19(\mathrm{~m}, 4 \mathrm{H}), 3.80(\mathrm{~d}, J$ $=10.0 \mathrm{~Hz}, 1 \mathrm{H}), 3.84(\mathrm{dd}, J=10.0,2.8 \mathrm{~Hz}, 1 \mathrm{H}), 6.87(\mathrm{~d}, J=$ $9.2 \mathrm{~Hz}, 1 \mathrm{H}), 6.90-7.00(\mathrm{~m}, 4 \mathrm{H}), 8.05(\mathrm{dd}, J=8.8,2.8 \mathrm{~Hz}$, $1 \mathrm{H}), 8.47(\mathrm{~d}, J=2.4 \mathrm{~Hz}, 1 \mathrm{H}) ;{ }^{13} \mathrm{C}$ NMR $\left(100 \mathrm{MHz}, \mathrm{CDCl}_{3}\right)$ $\delta$ 18.7, 26.7, 49.8, 51.6, 63.1, 70.3, 79.9, $115.6\left(\mathrm{~d}, J_{\mathrm{CF}}=22.0\right.$ $\mathrm{Hz}), 118.1,118.3\left(J_{\mathrm{CF}}=7.6 \mathrm{~Hz}\right), 122.7,124.8\left(\mathrm{~d}, J_{\mathrm{CF}}=10.4\right.$ $\mathrm{Hz}), 141.4\left(J_{\mathrm{CF}}=9.8 \mathrm{~Hz}\right), 148.0,153.2,158.6,159.4 ; \mathrm{MS}$ (EI) $m / z$ : $401\left(\mathrm{M}^{+}, 9 \%\right), 330$ (18), 179 (8), 150 (100), 136 (20), $122(20), 56(10)$.

$(3 R, 4 S)-4-(4-(2-M e t h o x y p h e n y l) p i p e r a z i n-1-y l)-2,2$ -dimethyl-6-nitrochroman-3-ol (1d): A yellow crystal, $\mathrm{mp}$ $153-154{ }^{\circ} \mathrm{C} ;[\alpha]_{D}^{20}-102.1\left(c 1.0, \mathrm{CH}_{2} \mathrm{Cl}_{2}\right)$; IR $(\mathrm{KBr}) v / \mathrm{cm}^{-1}$ : $3434,3074,2937,2833,1615,1584,1518,1500,1478$, $1375,1339,1270,1240,1138,1125,1075,1028,1014,996$, 944, 924, 838, 821, 793, 750, 696; ${ }^{1} \mathrm{H}$ NMR $(400 \mathrm{MHz}$, $\left.\mathrm{CDCl}_{3}\right) \delta 1.25(\mathrm{~s}, 3 \mathrm{H}), 1.55(\mathrm{~s}, 3 \mathrm{H}), 2.67(\mathrm{~s}, 1 \mathrm{H}), 3.01-3.11$ $(\mathrm{m}, 8 \mathrm{H}), 3.78-3.85(\mathrm{~m}, 2 \mathrm{H}), 3.87(\mathrm{~s}, 3 \mathrm{H}), 6.85-7.04(\mathrm{~m}, 5 \mathrm{H})$, $8.04(\mathrm{dd}, J=8.8,2.8 \mathrm{~Hz}, 1 \mathrm{H}), 8.51(\mathrm{~d}, J=2.8 \mathrm{~Hz}, 1 \mathrm{H}) ;{ }^{13} \mathrm{C}$ NMR $\left(100 \mathrm{MHz}, \mathrm{CDCl}_{3}\right) \delta 18.7,26.8,50.0,51.9,55.4,63.2$, $70.3,79.9,111.2,118.0,118.4,121.0,123.0,123.2,124.7$, 125.0, 141.1, 141.5, 152.2, 159.4; MS (EI) $m / z: 413\left(\mathrm{M}^{+}, 21\right.$ \%), 342 (18), 207 (11), 191 (14), 162 (100), 148 (25), 134
(19), 120 (11), 56 (12).

$(3 R, 4 S)-4-(4-(3-M e t h o x y p h e n y l) p i p e r a z i n-1-y l)-2,2-$ dimethyl-6-nitrochroman-3-ol (1e): A light-yellow solid, mp 84-87 ${ }^{\circ} \mathrm{C} ;[\alpha]_{D}^{20}-152.3\left(c 1.0, \mathrm{CH}_{2} \mathrm{Cl}_{2}\right)$; IR $(\mathrm{KBr}) v / \mathrm{cm}^{-1}$ : $3435,3095,3079,2989,2970,2944,2853,2832,1614$, $1576,1509,1476,1448,1427,1383,1333,1297,1274$, $1218,1204,1170,1150,1126,1072,1059,997,940,839$, 751, 698, 680; ${ }^{1} \mathrm{H}$ NMR $\left(400 \mathrm{MHz}, \mathrm{CDCl}_{3}\right) \delta 1.25(\mathrm{~s}, 3 \mathrm{H})$, $1.54(\mathrm{~s}, 3 \mathrm{H}), 2.61(\mathrm{~d}, J=2.4 \mathrm{~Hz}, 1 \mathrm{H}), 2.97-3.07(\mathrm{~m}, 4 \mathrm{H})$, 3.18-3.27 (m, 4H), $3.79(\mathrm{~d}, J=10.0 \mathrm{~Hz}, 1 \mathrm{H}), 3.80(\mathrm{~s}, 3 \mathrm{H})$, $3.83(\mathrm{dd}, J=10.0,2.8 \mathrm{~Hz}, 1 \mathrm{H}), 6.44(\mathrm{dd}, J=8.0,2.0 \mathrm{~Hz}$, $1 \mathrm{H}), 6.48(\mathrm{t}, J=2.4 \mathrm{~Hz}, 1 \mathrm{H}), 6.56(\mathrm{dd}, J=8.0,2.0 \mathrm{~Hz}, 1 \mathrm{H})$, $6.87(\mathrm{~d}, J=8.8 \mathrm{~Hz}, 1 \mathrm{H}), 7.18(\mathrm{t}, J=8.0 \mathrm{~Hz}, 1 \mathrm{H}), 8.05(\mathrm{dd}, J$ $=8.8,2.4 \mathrm{~Hz}, 1 \mathrm{H}), 8.47(\mathrm{~d}, J=2.0 \mathrm{~Hz}, 1 \mathrm{H}) ;{ }^{13} \mathrm{C} \mathrm{NMR}(100$ $\left.\mathrm{MHz}, \mathrm{CDCl}_{3}\right) \delta 18.7,26.8,49.8,50.5,55.2,63.1,70.3,79.9$, $102.9,104.9,109.2,118.1,122.7,124.7,124.9,129.8$, 141.4, 152.7, 159.4, 160.6; MS (EI) $m / z: 413\left(\mathrm{M}^{+}, 23 \%\right)$, 342 (12), 281 (16), 207 (33), 191 (15), 162 (100), 151 (23), 135 (17), 56 (11).

$(3 R, 4 S)-4-(4-(4-M e t h o x y p h e n y l) p i p e r a z i n-1-y l)-2,2-$ dimethyl-6-nitrochroman-3-ol (1f): An off-white solid, mp $183-184^{\circ} \mathrm{C}$; IR $(\mathrm{KBr}) v / \mathrm{cm}^{-1}: 3336,3073,3038,2979$, 2948, 2930, 2896, 2833,1613,1584, 1509, 1463, 1407, $1370,1348,1333,1292,1270,1251,1220,1178,1165$, $1137,1062,1033,1016,993,945,927,914,827,747,712$, 691; $[\alpha]_{D}^{20}-170.2\left(c 1.0, \mathrm{CH}_{2} \mathrm{Cl}_{2}\right) ;{ }^{1} \mathrm{H}$ NMR $(400 \mathrm{MHz}$, $\left.\mathrm{CDCl}_{3}\right) \delta 1.24(\mathrm{~s}, 3 \mathrm{H}), 1.49(\mathrm{~s}, 3 \mathrm{H}), 2.86(\mathrm{~s}, 1 \mathrm{H}), 3.02-3.06$ $(\mathrm{m}, 4 \mathrm{H}), 3.11-3.13(\mathrm{~m}, 4 \mathrm{H}), 3.78(\mathrm{~s}, 3 \mathrm{H}), 3.81-3.85(\mathrm{~m}, 2 \mathrm{H})$, 6.84-6.94 (m, 5H), 8.04 (dd, $J=8.8,2.8 \mathrm{~Hz}, 1 \mathrm{H}), 8.48(\mathrm{~d}, J$ $=2.8 \mathrm{~Hz}, 1 \mathrm{H}) ;{ }^{13} \mathrm{C} \mathrm{NMR}\left(100 \mathrm{MHz}, \mathrm{CDCl}_{3}\right) \delta 18.6,26.8$, 49.9, 52.1, 55.6, 63.1, 70.2, 80.0, 114.4, 118.0, 118.7, 122.8, 124.7, 124.9, 141.4, 145.6, 154.1, 159.4; MS (EI) $m / z: 413$ $\left(\mathrm{M}^{+}, 32 \%\right), 355(8), 341$ (7), 327 (8), 281 (47), 267 (7), 253 (18), 207 (100), 191 (24), 162 (62), 152 (15), 135 (22), 120 (12), 96 (11), 73 (10), 59 (10), 44 (31).

(3R,4S)-2,2-Dimethyl-6-nitro-4-(4-(3-(trifluoromethyl) phenyl)piperazin-1-yl)chroman-3-ol (1g): A light-yellow solid, mp 76-79 ${ }^{\circ} \mathrm{C}$; IR (KBr) v/cm ${ }^{-1}: 3434,3055,2978$, 2936, 2837, 1614, 1586, 1517, 1450, 1323, 1271, 1166, $1124,1075,1013,994,947,837,787,751,696 ;[\alpha]_{D}^{20}-$ 143.6 (c 1.0, $\mathrm{CH}_{2} \mathrm{Cl}_{2}$ ); ${ }^{1} \mathrm{H}$ NMR (400 MHz, $\mathrm{CDCl}_{3}$ ) $\delta 1.17$ $(\mathrm{s}, 3 \mathrm{H}), 1.46(\mathrm{~s}, 3 \mathrm{H}), 2.53(\mathrm{~d}, J=2.0 \mathrm{~Hz}, 1 \mathrm{H}), 2.93-2.99(\mathrm{~m}$, $4 \mathrm{H}), 3.15-3.24(\mathrm{~m}, 4 \mathrm{H}), 3.74(\mathrm{~d}, J=10.0 \mathrm{~Hz}, 1 \mathrm{H}), 3.80(\mathrm{dd}$, $J=10.0,2.0 \mathrm{~Hz}, 1 \mathrm{H}), 6.79(\mathrm{~d}, J=9.2 \mathrm{~Hz}, 1 \mathrm{H}), 6.99-7.06(\mathrm{~m}$, $3 \mathrm{H}), 7.28(\mathrm{t}, J=8.0 \mathrm{~Hz}, 1 \mathrm{H}), 7.97(\mathrm{dd}, J=8.8,2.4 \mathrm{~Hz}, 1 \mathrm{H})$, $8.41(\mathrm{~d}, J=2.0 \mathrm{~Hz}, 1 \mathrm{H}) ;{ }^{13} \mathrm{C}$ NMR $\left(100 \mathrm{MHz}, \mathrm{CDCl}_{3}\right) \delta$ 18.9, 26.7, 49.7, 50.1, 63.1, 70.5, 79.9, 112.6, 113.4, 116.2, $118.4,119.2,121.2,124.7,124.8\left(\mathrm{~d}, J_{\mathrm{CF}}=3.1 \mathrm{~Hz}\right), 129.6$, 136.2, 164.8, 167.7, 172.0; MS (EI) $m / z$ : 451 ( $\left.\mathrm{M}^{+}, 9 \%\right), 380$ (22), 200 (100), 172 (17), 145 (8), 56 (8).

(3S,4R)-2,2-Dimethyl-6-nitro-4-(4-phenylpiperazin-1yl)chroman-3-ol (2a): A white solid, mp $161-162{ }^{\circ} \mathrm{C}$; $[\alpha]_{D}^{20}+127.0\left(c 1.0, \mathrm{CH}_{2} \mathrm{Cl}_{2}\right)$; IR (KBr) $v / \mathrm{cm}^{-1}: 3391,3081$, $3027,3012,2980,2948,2913,2888,2820,1602,1586$, $1527,1496,1479,1426,1384,1371,1341,1270,1224$, 1167, 1137, 1069, 1012, 996, 942, 914, 839, 769, 747, 737, 695; ${ }^{1} \mathrm{H}$ NMR (400 MHz, $\left.\mathrm{CDCl}_{3}\right) \delta 1.24(\mathrm{~s}, 3 \mathrm{H}), 1.51(\mathrm{~s}$, 
$3 \mathrm{H}), 2.74(\mathrm{~d}, J=2.4 \mathrm{~Hz}, 1 \mathrm{H}), 2.99-3.07$ (m, 4H), 3.19-3.27 $(\mathrm{m}, 4 \mathrm{H}), 3.80(\mathrm{~d}, J=10.0 \mathrm{~Hz}, 1 \mathrm{H}), 3.84(\mathrm{dd}, J=10.0,2.4$ $\mathrm{Hz}, 1 \mathrm{H}), 6.85-6.96$ (m, 4H), 7.26-7.30 (m, 2H), 8.04 (dd, $J=$ 9.2, $2.8 \mathrm{~Hz}, 1 \mathrm{H}), 8.48(\mathrm{~d}, J=2.4 \mathrm{~Hz}, 1 \mathrm{H}) ;{ }^{13} \mathrm{C} \mathrm{NMR}(100$ $\left.\mathrm{MHz}, \mathrm{CDCl}_{3}\right) \delta 18.7,26.7,49.8,50.7,63.1,70.2,79.9$, 116.5, 118.0, 120.2, 122.8, 124.7, 124.9, 129.1, 141.4, 151.3, 159.4; MS (EI) $m / z: 383\left(\mathrm{M}^{+}, \%\right), 312$ (20), 161 (11), 132 (100), $118(24), 104(8), 77$ (10), 56 (11).

$(3 S, 4 R)-4-(4-(2-F l u o r o p h e n y l) p i p e r a z i n-1-y l)-2,2-$ dimethyl-6-nitrochroman-3-ol (2b): A light-yellow solid, mp 83-86 ${ }^{\circ} \mathrm{C} ;[\alpha]_{D}^{20}+99.7\left(c 1.0, \mathrm{CH}_{2} \mathrm{Cl}_{2}\right)$; IR $(\mathrm{KBr}) \mathrm{v} / \mathrm{cm}^{-1}$ : $3435,3075,2977,2937,2850,2829,1646,1314,1583$, $1517,1500,1478,1452,1428,1378,1339,1271,1238$, 1207, 1168, 1137, 1074, 1013, 996, 945, 926, 839, 810, 751, 695; ${ }^{1} \mathrm{H}$ NMR (400 MHz, $\left.\mathrm{CDCl}_{3}\right) \delta 1.25(\mathrm{~s}, 3 \mathrm{H}), 1.54(\mathrm{~s}$, $3 \mathrm{H}), 2.62(\mathrm{~d}, J=2.8 \mathrm{~Hz}, 1 \mathrm{H}), 3.02-3.10(\mathrm{~m}, 4 \mathrm{H}), 3.12-3.18$ $(\mathrm{m}, 4 \mathrm{H}), 3.79(\mathrm{~d}, J=10.0 \mathrm{~Hz}, 1 \mathrm{H}), 3.87$ (dd, $J=10.0,3.2$ $\mathrm{Hz}, 1 \mathrm{H}), 6.86(\mathrm{~d}, J=9.2 \mathrm{~Hz}, 1 \mathrm{H}), 6.93-7.10(\mathrm{~m}, 4 \mathrm{H}), 8.05$ $(\mathrm{dd}, J=9.2,2.8 \mathrm{~Hz}, 1 \mathrm{H}), 8.51(\mathrm{~d}, J=2.4 \mathrm{~Hz}, 1 \mathrm{H}) ;{ }^{13} \mathrm{C} \mathrm{NMR}$ $\left(100 \mathrm{MHz}, \mathrm{CDCl}_{3}\right) \delta 18.7,26.8,49.8,51.6,63.1,70.3,79.9$, $116.1\left(\mathrm{~d}, J_{\mathrm{CF}}=20.5 \mathrm{~Hz}\right), 118.0,119.2,122.7\left(\mathrm{~d}, J_{\mathrm{CF}}=7.7\right.$ $\mathrm{Hz}), 122.9,124.5,124.8\left(\mathrm{~d}, J_{\mathrm{CF}}=22.3 \mathrm{~Hz}\right), 140.0\left(\mathrm{~d}, J_{\mathrm{CF}}=\right.$ $8.3 \mathrm{~Hz}), 141.5,154.5,157.0,159.4$; MS (EI) $\mathrm{m} / \mathrm{z}$ : $401\left(\mathrm{M}^{+}\right.$, 14\%), 330 (26), 150 (100), 136 (26), 122 (27), 56 (12).

(3S,4R)-4-(4-(4-Fluorophenyl)piperazin-1-yl)-2,2dimethyl-6-nitrochroman-3-ol (2c): A light-yellow solid, mp 156-158 ${ }^{\circ} \mathrm{C} ;[\alpha]_{D}^{20}+138.1\left(c 1.0, \mathrm{CH}_{2} \mathrm{Cl}_{2}\right)$; IR (KBr) v/ $\mathrm{cm}^{-1}: 3464,3096,3055,2976,2945,2899,2879,2836$, $2810,1612,1584,1511,1483,1455,1427,1412,1384$, $1370,1338,1319,1263,1233,1221,1170,1136,1094$, 1064, 1008, 993, 985, 947, 916, 836, 762, 749, 718, 707, 688; ${ }^{1} \mathrm{H}$ NMR (400 MHz, $\left.\mathrm{CDCl}_{3}\right) \delta 1.25(\mathrm{~s}, 3 \mathrm{H}), 1.54(\mathrm{~s}$, $3 \mathrm{H}), 2.62(\mathrm{~d}, J=2.4 \mathrm{~Hz}, 1 \mathrm{H}), 2.98-3.09(\mathrm{~m}, 4 \mathrm{H}), 3.11-3.19$ $(\mathrm{m}, 4 \mathrm{H}), 3.79-3.86(\mathrm{~m}, 2 \mathrm{H}), 6.86-7.00(\mathrm{~m}, 5 \mathrm{H}), 8.05(\mathrm{dd}, J=$ $8.8,2.8 \mathrm{~Hz}, 1 \mathrm{H}), 8.47(\mathrm{~d}, J=2.4 \mathrm{~Hz}, 1 \mathrm{H}) ;{ }^{13} \mathrm{C}$ NMR $(100$ $\left.\mathrm{MHz}, \mathrm{CDCl}_{3}\right) \delta 18.7,26.8,49.8,51.6,63.1,70.3,79.9$, $115.6\left(\mathrm{~d}, J_{\mathrm{CF}}=22.0 \mathrm{~Hz}\right), 118.1,118.3\left(J_{\mathrm{CF}}=7.6 \mathrm{~Hz}\right), 122.7$, $124.8\left(\mathrm{~d}, J_{\mathrm{CF}}=10.4 \mathrm{~Hz}\right), 141.4\left(J_{\mathrm{CF}}=9.8 \mathrm{~Hz}\right), 148.0,153.2$, 158.6, 159.4; MS (EI) m/z: $401\left(\mathrm{M}^{+}, 17 \%\right), 330$ (17), 179 (7), 150 (100), $136(21), 122$ (21), 56 (10).

(3S,4R)-4-(4-(2-Methoxyphenyl)piperazin-1-yl)-2,2dimethyl-6-nitrochroman-3-ol (2d): A light-yellow solid, mp 83-86 ${ }^{\circ} \mathrm{C}$. $[\alpha]_{D}^{20}+108.0\left(c 1.0, \mathrm{CH}_{2} \mathrm{Cl}_{2}\right)$; IR (KBr) $v / \mathrm{cm}^{-1}$ : 3398, 3077, 2972, 2942, 2916, 2839, 2819, 1617, 1584, $1519,1500,1478,1374,1345,1266,1241,1168,1133,1124$, $1077,1046,1032,996,944,923,838,749,719,694 ;{ }^{1} \mathrm{H}$ NMR (400 MHz, $\left.\mathrm{CDCl}_{3}\right) \delta 1.25(\mathrm{~s}, 3 \mathrm{H}), 1.54(\mathrm{~s}, 3 \mathrm{H}), 2.69$ $(\mathrm{s}, 1 \mathrm{H}), 3.03-3.11(\mathrm{~m}, 8 \mathrm{H}), 3.78-3.85(\mathrm{~m}, 2 \mathrm{H}), 3.87(\mathrm{~s}, 3 \mathrm{H})$, 6.85-7.04 (m, 5H), $8.04(\mathrm{dd}, J=9.2,2.8 \mathrm{~Hz}, 1 \mathrm{H}), 8.51(\mathrm{~d}, J$ $=2.4 \mathrm{~Hz}, 1 \mathrm{H}) ;{ }^{13} \mathrm{C} \mathrm{NMR}\left(100 \mathrm{MHz}, \mathrm{CDCl}_{3}\right) \delta 18.7,26.8$, 50.0, 51.9, 55.4, 63.2, 70.3, 79.9, 111.2, 118.0, 118.4, 121.0, 123.0, 123.2, 124.7, 125.0, 141.1, 141.5, 152.2, 159.4; MS (EI) $m / z$ : $413\left(\mathrm{M}^{+}, 22 \%\right), 342$ (14), 207 (8), 191 (14), 162 (100), 148 (25), 134 (19), 120 (10), 56 (11).

(3S,4R)-4-(4-(3-Methoxyphenyl)piperazin-1-yl)-2,2dimethyl-6-nitrochroman-3-ol (2e): A light-yellow solid, mp 84-87 ${ }^{\circ} \mathrm{C} ;[\alpha]_{D}^{20}+131.1\left(c 1.0, \mathrm{CH}_{2} \mathrm{Cl}_{2}\right)$; IR $(\mathrm{KBr}) v / \mathrm{cm}^{-1}$ :
3429, 3079, 2975, 2938, 2934, 1604, 1583, 1496, 1479, 1452, 1373, 1338, 1269, 1201, 1170, 1074, 150, 1013, 994, 943, 838, 750, 694; ${ }^{1} \mathrm{H}$ NMR (400 MHz, $\left.\mathrm{CDCl}_{3}\right) \delta 1.25(\mathrm{~s}$, $3 \mathrm{H}), 1.53(\mathrm{~s}, 3 \mathrm{H}), 2.65(\mathrm{~d}, J=2.4 \mathrm{~Hz}, 1 \mathrm{H}), 2.97-3.06(\mathrm{~m}$, $4 \mathrm{H}), 3.17-3.27(\mathrm{~m}, 4 \mathrm{H}), 3.80(\mathrm{~d}, J=10.0 \mathrm{~Hz}, 1 \mathrm{H}), 3.80(\mathrm{~s}$, $3 \mathrm{H}), 3.83(\mathrm{dd}, J=10.0,2.0 \mathrm{~Hz}, 1 \mathrm{H}), 6.44(\mathrm{dd}, J=8.0,2.4$ $\mathrm{Hz}, 1 \mathrm{H}), 6.48(\mathrm{t}, J=2.4 \mathrm{~Hz}, 1 \mathrm{H}), 6.55(\mathrm{dd}, J=8.0,2.0 \mathrm{~Hz}$, $1 \mathrm{H}), 6.87(\mathrm{~d}, J=8.8 \mathrm{~Hz}, 1 \mathrm{H}), 7.18(\mathrm{t}, J=8.0 \mathrm{~Hz}, 1 \mathrm{H}), 8.05$ $(\mathrm{dd}, J=9.2,2.8 \mathrm{~Hz}, 1 \mathrm{H}), 8.47(\mathrm{~d}, J=2.4 \mathrm{~Hz}, 1 \mathrm{H}) ;{ }^{13} \mathrm{C} \mathrm{NMR}$ $\left(100 \mathrm{MHz}, \mathrm{CDCl}_{3}\right) \delta 18.7,26.8,49.8,50.5,55.2,63.1,70.3$, 79.9, 102.9, 104.9, 109.2, 118.1, 122.7, 124.7, 124.9, 129.8, 141.4, 152.7, 159.4, 160.6; MS (EI) m/z: $413\left(\mathrm{M}^{+}, \%\right), 342$ (17), 281 (11), 207 (25), 191 (15), 162 (100), 151 (22), 135 (11), $56(9)$.

(3S,4R)-4-(4-(4-Methoxyphenyl)piperazin-1-yl)-2,2dimethyl-6-nitrochroman-3-ol (2f): A light-yellow solid, mp 143-144 ${ }^{\circ} \mathrm{C}$; $[\alpha]_{D}^{20}+133.9\left(c 1.0, \mathrm{CH}_{2} \mathrm{Cl}_{2}\right)$; IR (KBr) v/ $\mathrm{cm}^{-1}:$ 3390, 3074, 3039, 2978, 2948, 2895, 2948, 2833, $1614,1585,1510,1463,1406,1379,1370,1348,1334$, $1270,1251,1221,1179,1166,1137,1077,1063,1034$, $1016,993,945,927,914,827,747,729,712,692,633 ;{ }^{1} \mathrm{H}$ NMR (400 MHz, $\left.\mathrm{CDCl}_{3}\right) \delta 1.24(\mathrm{~s}, 3 \mathrm{H}), 1.50(\mathrm{~s}, 3 \mathrm{H}), 2.86$ (s, 1H), 3.00-3.06 (m, 4H), 3.11-3.13 (m, 4H), $3.78(\mathrm{~s}, 3 \mathrm{H})$, 3.81-3.85 (m, 2H), 6.84-6.94 (m, 5H), 8.04 (dd, $J=9.2,2.0$ $\mathrm{Hz}, 1 \mathrm{H}), 8.48(\mathrm{~d}, J=2.0 \mathrm{~Hz}, 1 \mathrm{H}) ;{ }^{13} \mathrm{C}$ NMR $(100 \mathrm{MHz}$, $\left.\mathrm{CDCl}_{3}\right) \delta 18.6,26.7,49.9,52.1,55.6,63.0,70.2,79.9,114.4$, $118.0,118.7,122.8,124.7,124.9,141.4,145.6,154.0$, 159.4; MS (EI) m/z: $413\left(\mathrm{M}^{+}, 43 \%\right), 342$ (10), 191 (21), 162 (100), 148 (22), 134 (21), 120 (16), 56 (10).

(3S,4R)-2,2-Dimethyl-6-nitro-4-(4-(3-(trifluoromethyl) phenyl)piperazin-1-yl)chroman-3-ol (2g): A light-yellow solid, mp 74-76 ${ }^{\circ} \mathrm{C}$; $[\alpha]_{D}^{20}+140.0\left(c 1.0, \mathrm{CH}_{2} \mathrm{Cl}_{2}\right)$; IR (KBr) $v / \mathrm{cm}^{-1}: 3428,2977,2937,2837,1613,1586,1517,1479$, 1450, 1323, 1271, 1238, 1166, 1125, 1076, 1049, 1013, 994, 947, 837, 788, 751, 697; ${ }^{1} \mathrm{H}$ NMR (400 MHz, $\left.\mathrm{CDCl}_{3}\right) \delta 1.26$ $(\mathrm{s}, 3 \mathrm{H}), 1.54(\mathrm{~s}, 3 \mathrm{H}), 2.53(\mathrm{~d}, J=3.2 \mathrm{~Hz}, 1 \mathrm{H}), 3.00-3.07(\mathrm{~m}$, $4 \mathrm{H}), 3.23-3.32(\mathrm{~m}, 4 \mathrm{H}), 3.82(\mathrm{~d}, J=2.8 \mathrm{~Hz}, 1 \mathrm{H}), 3.86(\mathrm{dd}, J$ $=10.0,2.8 \mathrm{~Hz}, 1 \mathrm{H}), 6.87(\mathrm{~d}, J=8.8 \mathrm{~Hz}, 1 \mathrm{H}), 7.07-7.13(\mathrm{~m}$, $3 \mathrm{H}), 7.36(\mathrm{t}, J=8.0 \mathrm{~Hz}, 1 \mathrm{H}), 8.05(\mathrm{dd}, J=8.8,2.4 \mathrm{~Hz}, 1 \mathrm{H})$, $8.48(\mathrm{~d}, J=2.0 \mathrm{~Hz}, 1 \mathrm{H}) ;{ }^{13} \mathrm{C} \mathrm{NMR}\left(100 \mathrm{MHz}, \mathrm{CDCl}_{3}\right)$ 818.7, 26.7, 49.7, 50.1, 63.1, 70.4, 79.9, 112.6, 113.4, 116.2, $118.1,119.2,121.2,124.7,124.8\left(\mathrm{~d}, J_{\mathrm{CF}}=3.1 \mathrm{~Hz}\right), 129.6$, 136.2, 164.8, 167.7, 172.0; MS (EI) $m / z$ : $451\left(\mathrm{M}^{+}, 8 \%\right), 380$ (20), 200 (100), 172 (11), 145 (9), 56 (9).

Acknowledgments. This work were supported by the Priority Research Centers Program through the National Research Foundation of Korea (NRF) funded by the Ministry of Education, Science and Technology (2010-0029634) and by Changwon National University in 2009-2010.

\section{References}

1. (a) Chng, B. L.; Ganesan, A. Bioorg. Med. Chem. Lett. 1997, 7, 1511. (b) Rogers, G. A.; Parsons, S. M.; Anderson, D. C.; Nilsson, L. M.; Bahr, B. A.; Kornreich, W. D.; Kaufman, R.; Jacobs, R. S.; Kirtman, B. J. Med. Chem. 1989, 32, 1217.

2. (a) Deyrup, J. A.; Moyer, C. L. J. Org. Chem. 1969, 34, 175. (b) 
Smith, J. G. Synthesis 1984, 629.

3. Chini, M.; Crotti, P.; Flippin, L. A.; Macchia, F. J. Org. Chem. 1991, 56, 7043 .

4. Chini, M.; Crotti, P.; Favero, L.; Macchia, F.; Pineschi, M. Tetrahedron 1994, 35, 433 .

5. Beaton, M.; Gani, D. Tetrahedron Lett. 1998, 39, 8549.

6. Serrano, P.; Llebaria, A.; Delgado, A. J. Org. Chem. 2002, 67, 7165 .

7. Olofsson, B.; Somfai, P. J. Org. Chem. 2002, 67, 8574.

8. Xue, W. M.; Kung, M. C.; Kozlov, A. I.; Popp, K. E.; Kung, H. H. Catal. Today 2003, 85, 219.

9. Pachón, L. D.; Gamez, P.; Van Brussel, J. J. M.; Reedijk, J. Tetrahedron Lett. 2003, 44, 6025.

10. Cepanec, I.; Litviæ, M.; Mikuldaš, H.; Bartolinèiè, A.; Vinkoviè, V. Tetrahedron 2003, 59, 2435.

11. Fringuelli, F.; Pizzo, F.; Tortoioli, S.; Vaccaro, L. J. Org. Chem. 2004, 69, 7745 .

12. Ollevier, T.; Lavie-Compin, G. Tetrahedron Lett. 2004, 45, 49.

13. Ollevier, T.; Lavie-Compin, G. Tetrahedron Lett. 2002, 43, 7891.

14. Babiè, A.; Sova, M.; Gobec, S.; Peèar, S. Tetrahedron Lett. 2006, 47, 1733.

15. Overman, L. E.; Flippin, L. A. Tetrahedron Lett. 1981, 22, 195.

16. Rodríguez, J. R.; Navarro, A. Tetrahedron Lett. 2004, 45, 7495.

17. Bartoli, G.; Bosco, M.; Carlone, A.; Locatelli, M.; Massaccesi, M.; Melchiorre, P.; Sambri, L. Org. Lett. 2004, 6, 2173.

18. Chakraborti, A. K.; Rudrawar, S.; Kondaskar, A. Eur. J. Org. Chem. 2004, 3597 .
19. Lindsay, K. B.; Pyne, S. G. Tetrahedron 2004, 60, 4173.

20. Thibeault, D.; Poirier, D. Synlett 2003, 8, 1192.

21. Williams, D. B. G.; Lawton, M. Tetrahedron Lett. 2006, 47, 6557.

22. Yarapathy, V. R.; Mekala, S.; Rao, B. V.; Tammishetti, S. Catal. Comm. 2006, 7, 466.

23. Mojtahedi, M. M.; Saeed Abaee, M.; Hamidi, V. Catal. Comm. 2007, 8, 1671

24. (a) Swamy, N. R.; Goud, T. V.; Reddy, S. M.; Krishnaiah, P.; Venkateswarlu, Y. Synth. Comm. 2004, 34, 727. (b) Chakraborti, A. K.; Kondaskar, A. Tetrahedron Lett. 2003, 44, 8315.

25. (a) Lindsay, K. B.; Pyne, S. G. J. Org. Chem. 2002, 67, 7774. (b) Lindström, U. M.; Somfai, P. Tetrahedron Lett. 1998, 39, 7173. (c) Lindsay, K. B.; Tang, M.; Pyne, S. G. Synlett. 2002, 5, 731.

26. Chakraborti, A. K.; Rudrawar, S.; Kondaskar, A. Org. Biomol. Chem. 2004, 2, 1277.

27. Chakraborti, A. K.; Kondaskar, A.; Rudrawar, S. Tetrahedron 2004, 60, 9085.

28. Yadav, J. S.; Reddy, B. V. S.; Basak, A. K.; Venkat Narasaiah, A. Tetrahedron Lett. 2003, 44, 1047 .

29. Harváth, A.; Skoda-Földes, R.; Mahó, S.; Berente, Z.; Kollár, L. Steroids 2006, 71, 706

30. Azizi, N.; Saidi, M. R. Org. Lett. 2005, 7, 3649.

31. Evans, J. M.; Fake, C. S.; Hamilton, T. C.; Poyser, R. H.; Watts, E. A. J. Med. Chem. 1983, 26, 1582.

32. Yoo, S.-E.; Yi, K. Y.; Lee, S.; Suh, J.; Kim, N.; Lee, B. H.; Seo, H. W.; Kim, S.-O.; Lee, D.-H.; Lim, H.; Shin, H. S. J. Med. Chem. 2001, 44, 4207. 